\title{
A sample-based approach to estimate the dynamic loads of components with nonlinear uncertain interfaces
}

\author{
E. Menga ${ }^{\text {a,b,* }}$, M.J. Sánchez ${ }^{\mathrm{b}}$, I. Romero ${ }^{\mathrm{b}, \mathrm{c}}$, S. Hernández $^{\mathrm{d}}$ \\ a Airbus Operations S.L., A. John Lennon S/N, 28906 Getafe, Spain \\ b Universidad Politécnica de Madrid, José Gutiérrez Abascal, 2, 28006 Madrid, Spain \\ ${ }^{c}$ IMDEA Materials Institute, Eric Kandel, 2, Tecnogetafe 28906 Getafe, Spain \\ d Universidade da Coruña, Campus Elviña s/n, A Coruña 15071, Spain
}

\begin{abstract}
A B S T R A C T
Predicting aircraft dynamics and vibration loads at components' interfaces is a key task for ensuring a robust design and development of the product. Usually, whereas in the case of isolate components the dynamic behavior can be predicted quite accurately, when several components are assembled through discontinuous junctions the predictiveness of a model decreases. The junctions, whose mechanical properties are seldom well characterized experimentally, often introduce nonlinearities in the loads' path. Additionally, their behavior is intrinsically uncertain and as a consequence, the dynamic response of the connected structures becomes stochastic.

We propose a sample-based approach which aims to cope with both aspects, nonlinearities and uncertainties, and can be split in two main tasks. First, the computational cost of each deterministic simulation is minimized considering that the global nonlinear behavior depends on localized sources of nonlinearities at the interfaces. Second, the uncertainties are propagated through the model by a non-intrusive method based on Sobol's low discrepancy design. Attention is paid to the global sensitivity indices, which are estimated by creating a meta-model based on Polynomial Chaos Expansion.

An industrial application considering an aircraft component whose dynamic behavior is affected by uncertain free-plays at its interfaces is
\end{abstract} presented.

Keywords:

Nonlinear dynamics

Dynamic modifications

Uncertainty

Global sensitivity

Free-plays

\section{Introduction}

Assembled structures in the aerospace industry are often connected through discontinuous and nonlinear junctions whose mechanical properties are seldom well characterized experimentally. From a modeling point of view, they are often reduced to a set of linear springs with some stiffness and damping values. It happens that, during the project, these values are reviewed, argued and fine-tuned to get a reasonable or expected dynamic response $[1,2]$. Local parameters are changed one at a time to see their effect on the outputs: the higher the number of input parameters, the more tedious the task is and the more complex the understanding of the results as well. In particular, interfaces play a key role in defining the dynamics of components and systems but considering properly their behavior can be a very complex task. This is

\footnotetext{
* Corresponding author.

E-mail addresses: edoardo.menga@airbus.com (E. Menga),
}

especially true for dynamic studies [3,4]. Their behavior, often nonlinear, is intrinsically uncertain and as consequence, making the dynamic response of the connected structures stochastic.

This work looks at the Uncertainty Quantification (UQ) process as a systematic approach to establish, quantitatively and qualitatively, how the uncertain behavior of interfaces among components affects the overall dynamics of a structure. In the UQ process we include also the Global Sensitivity Analysis (GSA), which allows to rank the input parameters according to their influence on the variance of the stochastic Quantities of Interest (QoIs). These QoIs are precisely those outputs which allow to understand and make decisions about the structure.

Some researchers have already investigated the uncertainty within the joints of assembled structures [5,6], as well as in the assembly and manufacture of certain structural applications such as micro-electromechanical systems [7]. Most pragmatic and industrial approaches to UQ are based on sampling the deterministic model according some specific computer design, i.e., the Monte Carlo method, in order to produce a cloud of output points, which can then be used to obtain sample-based statistics. In any case, 
but especially for computationally expensive simulations, the direct use of Finite Element (FE) models can be extremely timeconsuming. Hence, usually, a smaller set of simulations is used to generate first a meta-model, which is used to complete the UQ analysis $[8,9]$. However, when the dynamic response of a structure is affected by strong nonlinearities, for example when the system has free-plays or includes rubber-like materials, the number of simulations required to generate a reliable meta-model can be still considerably high. The main reason is that these types of nonlinearities affect the smoothness of the high-dimensional response surface, which often cases will present discontinuities.

\subsection{Outline of the work}

In light of the previous observations, and considering that often junctions are represented by lumped elements, the Structural Dynamic Modification Method (SDMM) [10,11] seems a sensible approach for reducing the computational cost of the simulations. The principle behind modification methods is to run the FE Model just once, extracting the modal information at the required degrees of freedom (dofs) and then to update the equations, expressed in modal coordinates, through a set of modifications. While a large body of literature exists in the field of linear modifications, works dealing with nonlinear modifications are significant fewer, i.e. [12, 13]. The formulation of the Nonlinear Structural Dynamic Modification Method (NL SDMM) followed in this work has been introduced by Menga and Hernández [14-16] and is presented in Section 2. It has been proven to be a reliable and efficient approach to reduce drastically the computation burden associated with one deterministic nonlinear simulation when the global dynamic behavior depends on localized sources of nonlinearity, which can be modeled via lumped elements, i.e., elements connecting pairs of dofs.

In the current work, the NL SDMM is used to produce efficiently the initial set of simulations which will be used for generating the meta-model. Then the surrogate, based on a multivariate Polynomial Chaos Expansion (PCE), can be used to generate a broader sample of output points and obtaining the sensitivity indices.

A brief review of the PCE meta-modeling technique and of variance-based Global Sensitivity Analysis (GSA) is given in Section 3. Also, the mathematical link between the coefficients of the polynomial expansion and the sensitivity indices is discussed, although in a non-exhaustive fashion. The interest reader can refer for example to the work of Saltelli and Ratto [17] in the context of GSA and to the publications of Sudret [18] in the field of PCE.

Section 4 describes an industrial application of the previous ideas, combined in a systematic approach. The example considers an aircraft component whose dynamic behavior is affected by freeplay interfaces, which are intrinsically uncertain.

Finally, Section 5 summarizes the main conclusions and results of this research and proposes some possible future work.

\section{Nonlinear structural dynamic modifications}

In this section the theory behind the NL SDMM is reviewed. The modal matrix and the eigenvalues are assumed to be available from the FE modal analysis of the model. Also, we consider that the only source of nonlinearity in the model is due to the interfaces between components. In this section we formulate the equation in the case of a nonlinear stiffness update, but the same approach can be followed for mass and damping modifications.

The initial stiffness of the spring elements used in the FE model for obtaining the modal properties is called underlying linear stiffness, whose value, with reference to the Fig. 1 , is denoted $k_{1}$. Starting from the modal information of the model, we want to update the dynamic equations to take into account the nonlinear stiffness

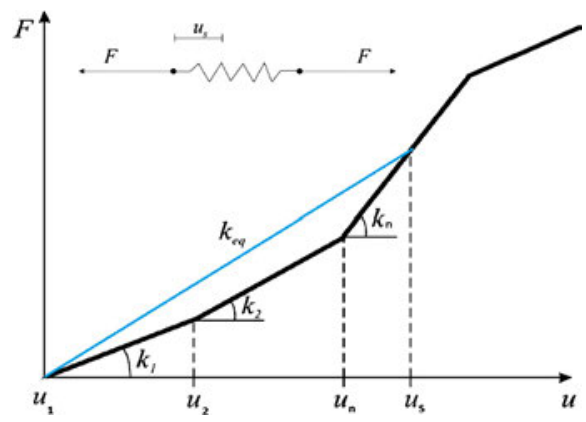

Fig. 1. Multi-linear force-displacement curve.

modifications. Then, the modal base of the linear model is fixed, i.e., the FE model is run just once, and the nonlinear response is the result of an updating process, irrespective of the type of nonlinearities that are incorporated.

The dynamic equilibrium in time-domain can be expressed as:

$M \ddot{u}(t)+C \dot{u}(t)+K u(t)=F(t)$,

where $M, C, K$ are, respectively, the mass, damping, and stiffness matrix, and $F$ is the vector of external forces. The eigenvalues and eigenvectors of the system are found by solving the problem:

$(K-\lambda M) \Phi=0$,

where $\lambda$ is a diagonal matrix with entries equal to the eigenvalues of the system, and $\Phi$ is the modal matrix. The $i$-th column of the modal matrix $\Phi$ is the eigenvector corresponding to the eigenvalue $\lambda_{i}$.

The displacements of the system can be expressed as a linear combination of the eigenvectors weighted by some factors $q(t)$, which are called modal coordinates, that is:

$u(t)=\Phi q(t)$.

When the modal matrix is normalized with the mass, Eq. (1) can be expressed in the well-known modal form:

$I \ddot{q}(t)+\tilde{C} \dot{q}(t)+\Lambda q(t)=\tilde{F}(t)$,

where $I, \tilde{C}$, and $\Lambda$ are now the normalized mass, damping and stiffness matrix, respectively, and $\tilde{F}$ is the vector of forces. In Eq. (4) the normalized mass matrix is the identity, and the normalized stiffness matrix is a diagonal matrix whose terms are the eigenvalues.

With reference to Fig. 1 , when a relative displacement $u_{s}$ is applied between the two dofs of the spring, according to the forcedisplacement curve, the internal force is:

$$
\begin{aligned}
F_{s}= & k_{1}\left(u_{2}-u_{1}\right)+k_{2}\left(u_{3}-u_{2}\right)+\ldots \\
& +k_{n-1}\left(u_{n}-u_{n-1}\right)+k_{n}\left(u_{s}-u_{n}\right) .
\end{aligned}
$$

The same force can be obtained with a secant stiffness given by:

$k_{\text {equ }}=\frac{\sum_{i=1}^{n-1} k_{i}\left(u_{i+1}-u_{i}\right)+k_{n}\left(u_{s}-u_{n}\right)}{u_{s}}$.

Considering an underlying linear stiffness value $k_{1}$, the additional contribution to the current stiffness is:

$\Delta k=k_{\text {equ }}-k_{1}$,

and the modification matrix is of the type:

$\Delta k_{\text {mod }}=\left[\begin{array}{cc}\Delta k & -\Delta k \\ -\Delta k & \Delta k\end{array}\right]$ 
When $N_{k}$ lumped elements are involved in the modification, the final matrix can be obtained as:

$\Delta K_{\text {mod }}=\sum_{m=1}^{N_{k}} \Delta k_{\text {mod }}^{m}$,

which can be normalized by the modal matrix of the underlying linear system:

$\Delta \tilde{K}_{\text {mod }}=\Phi^{T} \Delta K_{\text {mod }} \Phi$.

Hence, the update stiffness matrix of the structure is:

$\Lambda_{\text {mod }}=\Lambda+\Delta \tilde{K}_{\text {mod }}$,

and Eq. (4) can be re-written as:

$I \ddot{q}(t)+\tilde{C} \dot{q}(t)+\Lambda_{\bmod } q(t)=\tilde{F}(t)$.

Eq. (12) needs to be solved by an iterative method which has to take into account that $k_{\text {equ }}$, and consequently $\Lambda_{\text {mod }}$, are nonlinear functions of the relative displacements of the springs, which, through the modal matrix $\Phi$, depend on the modal displacements. In this work the state space form of Eq. (12) has been implemented in MATLAB ${ }^{\circledR}$ and solved with the function ODE45, which uses an explicit Runge-Kutta integration procedure. Once the nonlinear modal displacements are obtained, the physical ones, for all the dofs considered in the modal matrix, can be evaluated by Eq. (3). We stress the fact that this approach is computationally very efficient: the linear FE Model is run just once in order to obtain the modal matrix, and usually the number of significant modes is much smaller than the dofs of the model. Additionally, the dofs involved in the updating process are usually a few dozen: the dofs where the loads are applied, those ones where the outputs are required, and finally those ones involved in the modifications.

\section{Uncertainty quantification}

UQ can be thought as a process with three fundamental phases, namely, pre-processing, uncertainty propagation, and postprocessing. The first phase aims to select which input parameters are the source of uncertainties, and to prescribe on them some appropriate probability distribution. The propagation of the uncertainties aims to characterize the distributions of the Quantities of Interest (QoIs) for a given distribution of inputs. That is obtained, in non-intrusive methods, by sampling the deterministic model according to some specific sampling technique. Then, the cloud of results can be post-processed in order to obtain samplebased statistics. The most common design is based on Montecarlo (MC) sampling, which relies on pure randomness. Due its randomness, some points can be clustered closely, while other regions within the space may not contain any samples. Hence, especially when working in high dimensions, most researchers seem to agree that generic, near-uniform, distribution of the data sites is a better strategy. Several sampling strategies try to cover this need, such as the Latin Hypercube (LHD) and the Low-Discrepancy Design (LDD). LDD, in particular, generates a deterministic sequence of quasirandom numbers which fill uniformly the unit hypercube. In the context of this work, Sobol's Low Discrepancy Design (LDD) is considered [19]. It was the first digital sequence, operates in base-2, and has been found by several researchers, i.e. Pianosi [20], that this choice out-performs the classical MC and LHD methods being, in average, more efficient and having a faster convergence rate. For complex systems where, possibly, a very large set of inputs is considered uncertain, it is often necessary to generate first a meta-model and then, using it, complete the analysis.
Table 1

Correspondence between orthogonal polynomials and PDF.

\begin{tabular}{llll}
\hline Orthogonal polynomial & Weight function & PDF & Density function \\
\hline Hermite $H_{e}(x)$ & $e^{\frac{-x^{2}}{2}}$ & Normal & $\frac{1}{\sqrt{2 \pi}} e^{\frac{-x^{2}}{2}}$ \\
Legendre $P_{n}(x)$ & 1 & Uniform & $\frac{1}{2}$ \\
Laguerre $L_{n}(x)$ & $e^{-x}$ & Exponential & $e^{-x}$ \\
Jacobi $P_{n}^{\alpha, \beta}(x)$ & $x^{\alpha} e^{-x}$ & Beta & $\frac{x^{\alpha} e^{-x}}{\Gamma(\alpha+1)}$ \\
\hline
\end{tabular}

\subsection{Meta-model: polynomial chaos expansion}

The original Hermite Polynomial Chaos Expansion (PCE), also known as homogeneous chaos, was first derived by Wiener [21] for the spectral representation of any stochastic response in terms of Gaussian random variables. Later, Askey [22] studied the orthogonal properties of the Hermite polynomials with respect to the Gaussian probability density function, providing the well-known Wiener-Askey scheme. But only in 2003 Xiu [23] extended the method to others random distributions as summarised in Table 1. To ensure optimal convergence properties of the PCE, the polynomials are required to be orthogonal with respect to a weight function, which is representative of the probability distribution of the Quantities of Interest (QoIs).

To introduce the notation that will be employed later, let us briefly describe next the standard least square approximation problem: if a model is sampled at $N_{S}$ points of the high-dimensional space $\mathcal{S} \equiv \mathbb{R}^{N_{p}}$, the generic output $Y_{n}=Y\left(X_{n}\right)$ evaluated at the input point $X_{n}=\left[X_{n 1}, X_{n 2}, \ldots, X_{n N p}\right]$, with $n \in\left[1,2, \ldots, N_{s}\right]$ and $N_{p}$ the total number of input parameters considered, can be approximated by a linear combination of $N_{k}$ basis or kernel functions $\Psi_{k}: \mathcal{S} \rightarrow \mathbb{R}$ as

$Y\left(X_{n}\right) \simeq \sum_{k=1}^{N_{k}} \beta_{k} \Psi_{k}\left(u_{n}\right)$.

In our case, the basis functions are orthogonal polynomials with respect to a defined weight, and usually in as specific domain. For example, Legendre polynomials are orthogonal with respect a uniform probability density function in the domain $[-11]$. Hence, each $u_{n}$ is a normalised input random variable, and $X_{n}$ has to be mapped to the domain where the orthogonality is ensured.

This relation can be expressed more compactly using matrix notation as in

$Y(X) \simeq \Psi(u) \beta$,

where $\Psi$ is the interpolation or kernel matrix, $Y$ is the matrix of the $N_{\text {out }}$ QoIs evaluated at $N_{s}$ sampled points and $X$ is the sample matrix.

Usually, $\Psi_{k}$ are multivariate polynomials that involve products of the one-dimensional polynomials $\psi_{i}\left(u_{j}\right)$, where $i$ is the order of the polynomial and $u_{j}$ is the $j$-th component of the vector $u_{n}$. The multivariate expansion is obtained as a tensor product of the one-dimensional basis and consequently presents higher-order terms. These higher order terms are usually truncated considering for the multivariate basis the same maximum order 0 of the onedimensional polynomials they come from.

Under these conditions, the total number of coefficients $N_{k}$ of the polynomial expansion in Eq. (13) is given by:

$N_{k}=\frac{\left(N_{p}+0\right) !}{N_{p} ! o !}$.

As an example, we consider the first six one-dimensional Hermite polynomials: 
Table 2

Bivariate second order Hermite polynomials.

\begin{tabular}{llll}
\hline$\Psi_{0}(u)$ & $\psi_{0}\left(u_{1}\right) \cdot \psi_{0}\left(u_{2}\right)$ & $1 \cdot 1$ & 1 \\
$\Psi_{1}(u)$ & $\psi_{1}\left(u_{1}\right) \cdot \psi_{0}\left(u_{2}\right)$ & $u_{1} \cdot 1$ & $u_{1}$ \\
$\Psi_{2}(u)$ & $\psi_{0}\left(u_{1}\right) \cdot \psi_{1}\left(u_{2}\right)$ & $1 \cdot u_{2}$ & $u_{2}$ \\
$\Psi_{3}(u)$ & $\psi_{2}\left(u_{1}\right) \cdot \psi_{0}\left(u_{2}\right)$ & $\left(u_{1}^{2}-1\right) \cdot 1$ & $u_{1}^{2}-1$ \\
$\Psi_{4}(u)$ & $\psi_{0}\left(u_{1}\right) \cdot \psi_{2}\left(u_{2}\right)$ & $1 \cdot\left(u_{2}^{2}-1\right)$ & $u_{2}^{2}-1$ \\
$\Psi_{5}(u)$ & $\psi_{1}\left(u_{1}\right) \cdot \psi_{1}\left(u_{2}\right)$ & $u_{1} \cdot u_{2}$ & $u_{1} u_{2}$ \\
\hline
\end{tabular}

$$
\begin{aligned}
\{\psi(u)\}= & \left\{1, u, u^{2}-1, u^{3}-3 u,\right. \\
& \left.u^{4}-6 u^{2}+3, u^{5}-10 u^{3}+15 u, \ldots\right\},
\end{aligned}
$$

and the multivariate polynomials for a second-order expansion over two random variables, presented in Table 2 . The higher order terms, such as $\Psi_{6}(u)=\psi_{2}\left(u_{1}\right) \cdot \psi_{1}\left(u_{2}\right)=u_{1}^{2} u_{2}$, are neglected.

From Eq. (15) it can be noted that increasing the number of random variables or the order of the polynomial will cause a substantial increase in the number of terms $N_{k}$ of the PCE and the sample size $N_{S}$ required to find the coefficients of the expansion. Consequently, in the case of complex simulations, the computational cost could be unaffordable.

In this work the least square method is employed to estimate the unknown coefficients $\beta_{k}$ of the PCE. Considering Eq. (13) and minimizing the sum of the squares of the residuals:

$\beta=\arg \min _{\beta^{*} \in \mathbb{R}^{N_{k}}}\left\|\Psi \beta^{*}-Y\right\|^{2}$,

the least square fitting gives a closed-form solution:

$\beta=\left(\Psi^{T} \Psi\right)^{-1} \Psi^{T} Y$,

where $\Psi$ is assumed to have full column rank.

The number of input points $N_{s}$ must be higher than the number of unknown coefficients $N_{k}$ and, depending on the complexity of the response, the recommended number of samples may fluctuate. Following Hosder [24], $N_{s} \simeq 2 \cdot N_{k}$ has been employed.

\subsection{Global sensitivity analysis}

GSA defines a qualitative and quantitative mapping between the input variables and the Quantities of Interest (QoIs). Sensitivity analyses are strictly related to the Uncertainty Propagation (UP) and they can be seen as complementary to each other: UP quantifying the uncertainty in the output of the model, while GSA focuses on apportioning the output's uncertainty to the different sources of uncertain parameters [17].

GSA allows to identify the most relevant parameters of the model, and score them according to their relevance in the QoIs. In this context, a sensitivity index is a measure of the influence of an uncertain quantity on an output variable. The most popular GSA techniques are based on the decomposition of the variance's output probability distribution and allow the calculation of Sobol's sensitivity indices. They refer to Sobol', because the decomposition of the variance can be formulated analytically from the High Dimensional Model Representation (HDMR) of Sobol' [25]. From a practical point of view, particular attention is paid to the first order and total order sensitivity indices, denoted respectively as $S_{i}^{I}$ and $S_{i}^{T}$, also called the main effects and the total effects. If a model $Y$ is a function of $N_{p}$ stochastic variables $X_{i}$ with $i=1, \ldots, N_{p}$, the main effects represent the contribution of each parameter $X_{i}$ to the output variance, or equivalently, the expected percentage reduction of the output variance $V(Y)$ obtained when the uncertainty of input parameter $X_{i}$ is eliminated. The total effects represent the total contribution of each input parameter considering its individual effect and its interaction with the remaining factors.
Table 3

\begin{tabular}{|c|c|c|c|c|c|}
\hline \multirow{2}{*}{$\begin{array}{l}\text { Multivariate PCE } \\
\Psi_{0}(u)\end{array}$} & \multicolumn{3}{|c|}{ Multi-Index } & \multirow{2}{*}{$\begin{array}{l}\text { Coefficients } \\
\beta_{0}\end{array}$} & \multirow{2}{*}{$\begin{array}{l}\text { Function } \\
1\end{array}$} \\
\hline & $\alpha_{0}$ & 0 & 0 & & \\
\hline$\Psi_{1}(u)$ & $\alpha_{1}$ & 1 & 0 & $\beta_{1}$ & $u_{1}$ \\
\hline$\Psi_{2}(u)$ & $\alpha_{2}$ & 0 & 1 & $\beta_{2}$ & $u_{2}$ \\
\hline$\Psi_{3}(u)$ & $\alpha_{3}$ & 2 & 0 & $\beta_{3}$ & $u_{1}^{2}$ \\
\hline$\Psi_{4}(u)$ & $\alpha_{4}$ & 0 & 2 & $\beta_{4}$ & $u_{2}^{2}$ \\
\hline$\Psi_{5}(u)$ & $\alpha_{5}$ & 1 & 1 & $\beta_{5}$ & $u_{1} u_{2}$ \\
\hline
\end{tabular}

Multi-index: bivariate PCE of $2^{\text {nd }}$ order.

Table 4

Equivalence between Sobol's HDMR and PCE.

\begin{tabular}{llll}
\hline Sobol's HDMR & PCE functions & Variance & Variance PCE \\
\hline$f_{0}$ & $\beta_{0}$ & $V\left(f_{0}\right)$ & $\beta_{0}^{2}$ \\
$f_{1}$ & $\beta_{1} u_{1}+\beta_{3} u_{1}^{2}$ & $V\left(f_{1}\right)$ & $\beta_{1}^{2}+\beta_{3}^{2}$ \\
$f_{2}$ & $\beta_{2} u_{2}+\beta_{4} u_{2}^{2}$ & $V\left(f_{2}\right)$ & $\beta_{2}^{2}+\beta_{4}^{2}$ \\
$f_{3}$ & $\beta_{5} u_{1} u_{2}$ & $V\left(f_{3}\right)$ & $\beta_{5}^{2}$ \\
\hline
\end{tabular}

\subsection{Polynomial chaos and Sobol's indices}

According to the PCE theory, the mean and the variance of the QoIs can be estimated directly by means of the PCE coefficients. In fact, assuming that the polynomials are normalized with the variance, the following relationships hold:

$$
\begin{aligned}
& E(Y(X)) \approx \beta_{0}, \\
& V(Y(X)) \approx \sum_{k=1}^{N_{k}} \beta_{k}^{2} .
\end{aligned}
$$

In the context of multivariate polynomials, the concept of a multi-index $\alpha_{k}$ is often presented. This notation allows to describe the order of the multivariate terms from the tensor product of the univariate polynomials. Table 3 provides the multi-indices in the case of a second-order, bivariate PCE.

It is straightforward to see a close similarity between the PCE and the High Dimensional Model Reduction (HDMR) proposed by Sobol. In fact, the output functions can be seen as the sum of higher dimensional terms defined according the multi-index $\alpha_{k}$. In addition, Sobol's condition for the uniqueness of the decomposition requires expressing the output function as the sum of a constant term plus a higher dimensional, zero-mean function, a condition that is satisfied thanks to the orthogonality of the polynomials.

Continuing the previous example on a bivariate random variable, Sobol's HDMR considers the terms of increasing dimensionality:

$$
Y(X)=f\left(X_{1}, X_{2}\right)=f_{0}+f_{1}\left(X_{1}\right)+f_{2}\left(X_{2}\right)+f_{12}\left(X_{1}, X_{2}\right),
$$

while looking at the PCE, according to Table 3, the following expansion is obtained:

$$
Y(X) \simeq \beta_{0}+\beta_{1} u_{1}+\beta_{2} u_{2}+\beta_{3} u_{1}^{2}+\beta_{4} u_{2}^{2}+\beta_{5} u_{1} u_{2} .
$$

Comparing Eq. (21) and Eq. (22), it is possible to identify the equivalence between the two formulations, which allows an easier calculation of the partial variances and later of the sensitivity indices (Table 4).

In fact, the total variance, defined by Eq. (20) can be decomposed as:

$$
\begin{aligned}
V(Y) & =V\left(f_{0}\right)+V\left(f_{1}\right)+V\left(f_{2}\right)+V\left(f_{12}\right) \\
& =\beta_{0}^{2}+\left(\beta_{1}^{2}+\beta_{3}^{2}\right)+\left(\beta_{2}^{2}+\beta_{4}^{2}\right)+\beta_{5}^{2},
\end{aligned}
$$




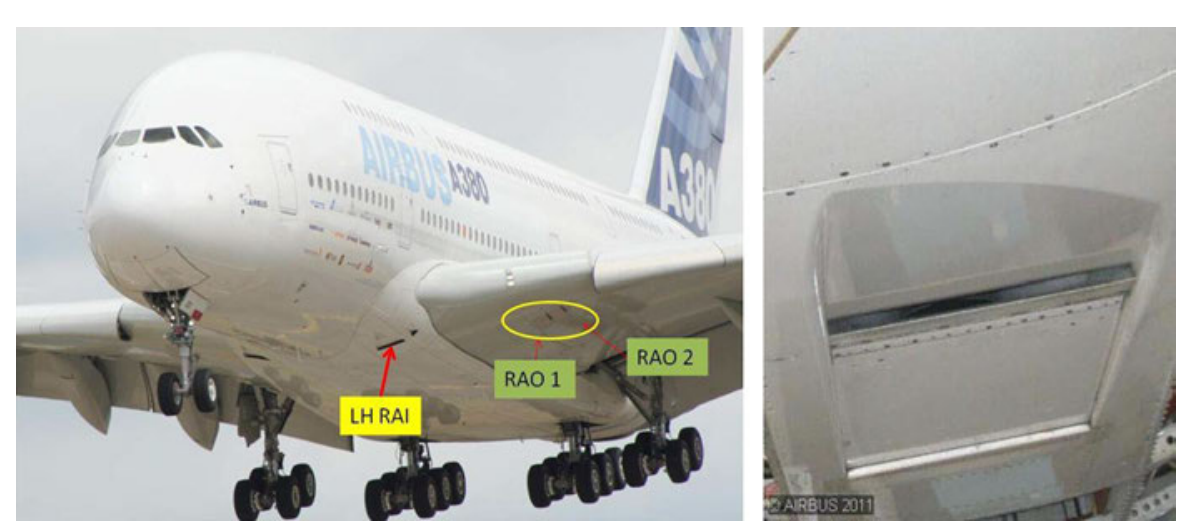

Fig. 2. A380 - RAI System and Air Intake detail (source|AIRBUS OPERATIONS).

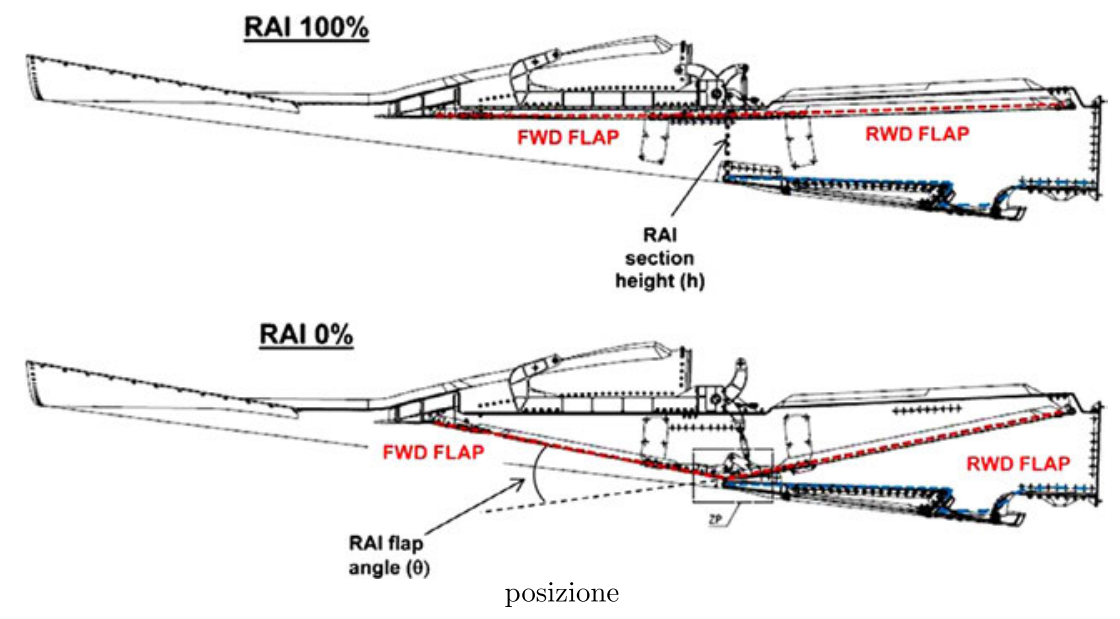

Fig. 3. A380 - RAI System Flaps (source|AIRBUS OPERATIONS).

and Sobol's indices can be obtained by the ratio between the partial variances and the total one. For example, the first and total effects with respect the first input variable $X_{1}$ are:

$S_{1}^{I}=\frac{V\left(f_{1}\right)}{V(Y)} \approx \frac{\beta_{1}^{2}+\beta_{3}^{2}}{\sum_{m=1}^{p} \beta_{m}^{2}}$,
$S_{1}^{T}=\frac{V\left(f_{1}\right)+V\left(f_{12}\right)}{V(Y)} \approx \frac{\beta_{1}^{2}+\beta_{3}^{2}+\beta_{5}^{2}}{\sum_{m=1}^{p} \beta_{m}^{2}}$.

Hence the calculation of the PCE coefficients provides a straightforward and computational inexpensive way to estimate the mean, the variance and Sobol's sensitivity indices. It is worth highlighting that Sobol's HDMR is an exact decomposition, while the polynomial expansion would be exact only if the summation were extended to infinite terms. In practice, the PCE is truncated to an order that in most applications does not exceed the third degree.

\section{Industrial application: A380 RAM Air Inlet free-play nonlinear vibrations}

In this section the UQ is considered in the context of an industrial case of study: A380 RAM Air Inlet nonlinear vibrations.

A RAM refers, in this context, to any moving part of a machine that exerts a force onto another one, by pushing or applying pressure on the latter. The RAM Air Inlet(RAI) and the RAM Air Outlet(RAO) openings provide a forced ventilation system to control the air temperature around the Air Generation Unit (AGU). This system supplies and maintains the air in the pressurised fuselage compartments at the correct pressure, temperature, and freshness for passenger comfort and equipment cooling. Also, it provides air for ventilation in the unpressurized fuselage bays. Fig. 2 shows, on the left, the Airbus A380 RAI air intake and two RAO outtakes. Air flow enters through the RAI intake, wets the forward and progressively the reward flaps, enables the AGU heat exchange by forced ventilation, and then comes out through the RAO outtakes.

This study focuses on the RAI system, shown in Fig. 3: The RAI forward (FWD) and rearward (RWD) flaps are two moving panels whose relative position defines the height of the RAI section and then the amount of air required for heat exchange. In Fig. 3 two extreme positions are shown: the maximum opening (RAI 100\%) and the minimum one (RAI 0\%). An actuator, through a kinematic chain, shown in Fig. 4, adjusts the RAI section height and consequently the flaps' relative position. 3D details of the kinematic chain can be seen in Fig. 5: the actuator drives the shaft, in green, and through two flap links, moves two rods (gray color) which regulate the height by acting on the RWD flap.

During a flight, the two flaps are subjected to an unsteady pressure field which induces vibrations in the RAI system. Additionally, due to tolerances and wear of the bearings, the connections between the flap links and the rods present free-plays, which make the dynamic problem and hence the rods' reaction forces, nonlinear. Free-play behavior is shown in Fig. 10 by a nonlinear forcedisplacement curve: the stiffness is zero when the dynamic relative displacements of the interfaces is within a certain tolerance $\delta$ and assumes a value given by Eq. (6) when they are out of this range. 

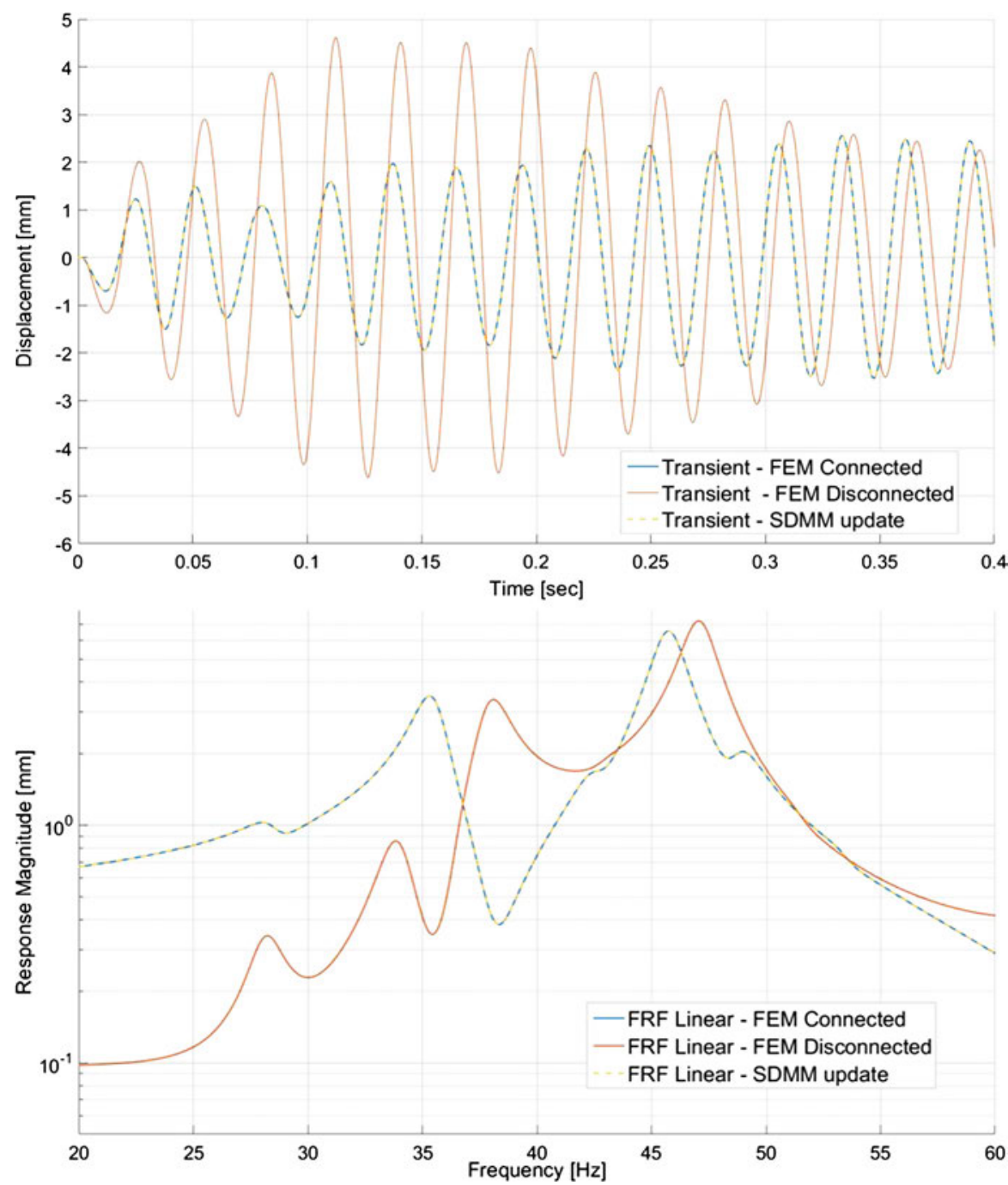

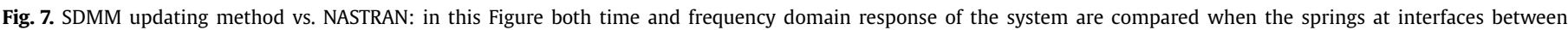

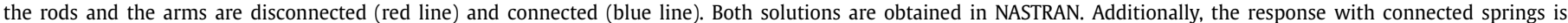

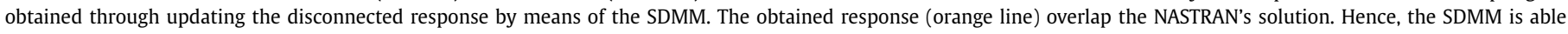
to consider disruptive change of local stiffnesses in both domains.

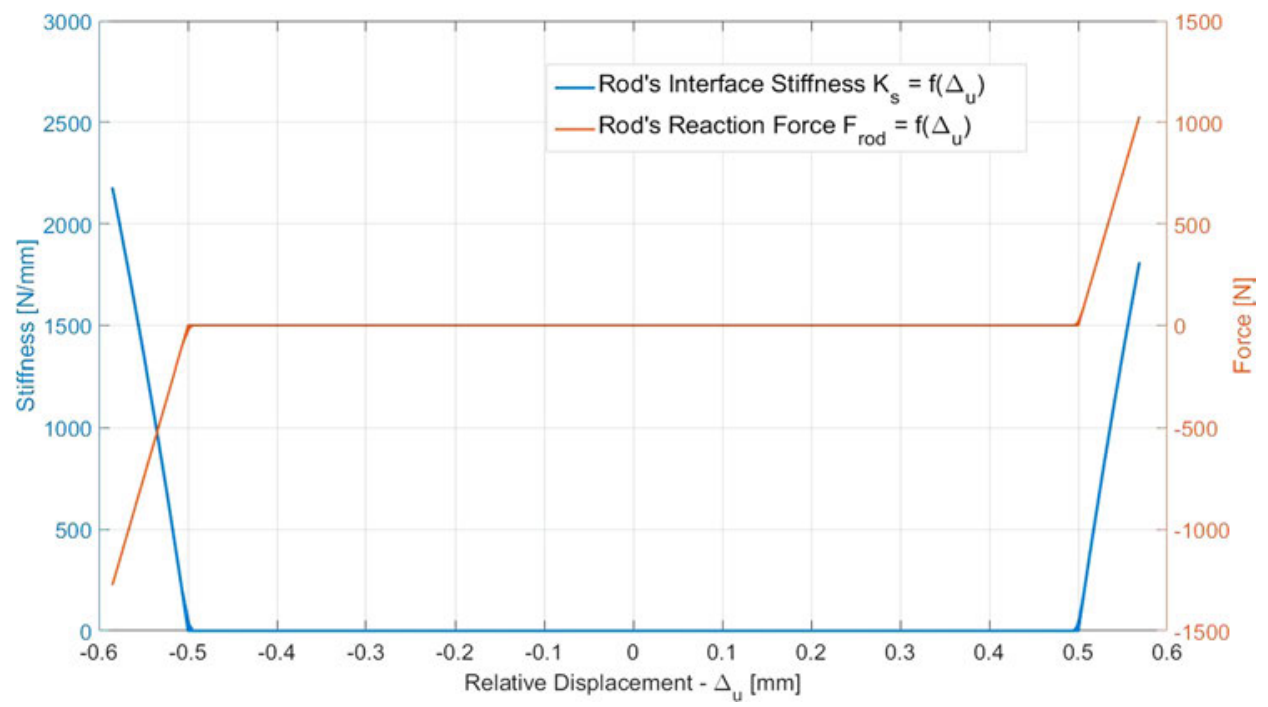

Fig. 8. Nonlinear stiffness and reaction force at nominal conditions as defined in Table 5 . 

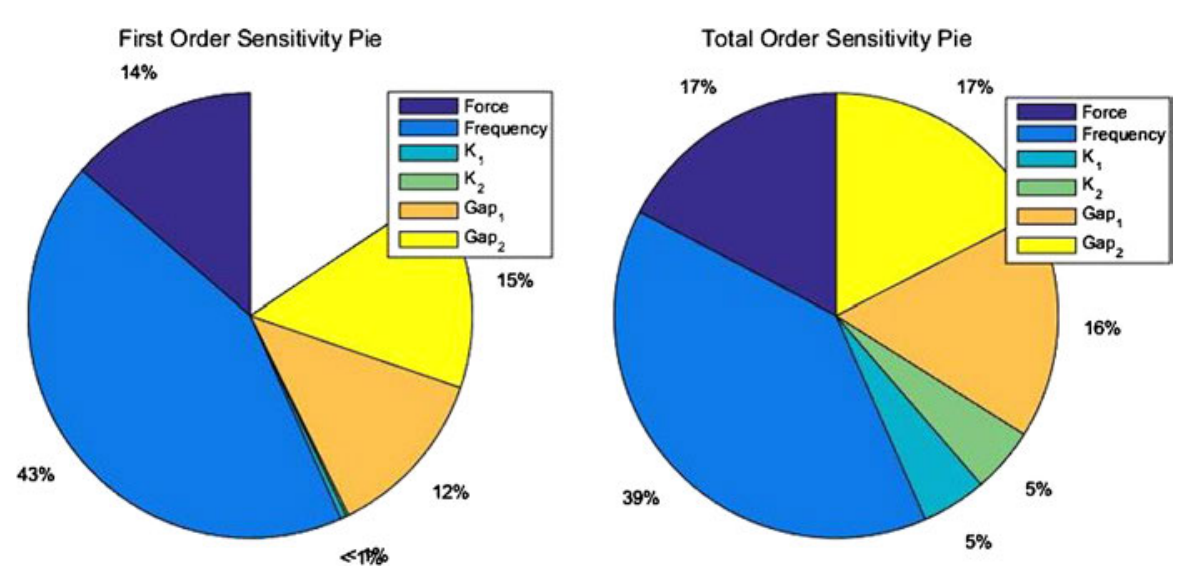

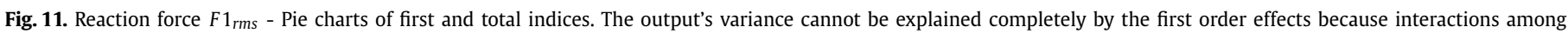

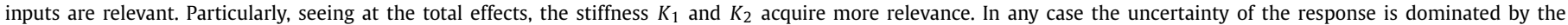
frequency of excitation first, and then by the magnitude of the applied force and by the size of the gaps of free-plays.

Table 6

RAI vibrations - statistical moments and Sobol's Indices.

\begin{tabular}{llll}
\hline Parameter & Results & $F 1_{\text {rms }}$ & $F 2_{r m s}$ \\
\hline & Mean(Y) & 140.10 & 207.49 \\
& V(Y) & 3286.55 & 8145.43 \\
& error on $N_{\text {eval }}$ & 0.0015 & 0.0024 \\
Force & $S_{1}$ & 0.14 & 0.17 \\
Frequency & $S_{2}$ & 0.43 & 0.30 \\
$K_{1}$ & $S_{3}$ & 0.00 & 0.01 \\
$K_{2}$ & $S_{4}$ & 0.00 & 0.01 \\
Gap & $S_{5}$ & 0.12 & 0.17 \\
Gap 2 & $S_{6}$ & 0.15 & 0.06 \\
Force & & & \\
Frequency & $S T_{1}$ & 0.21 & 0.28 \\
$K_{1}$ & $S T_{2}$ & 0.48 & 0.38 \\
$K_{2}$ & $S T_{3}$ & 0.06 & 0.10 \\
Gap $_{1}$ & $S T_{4}$ & 0.06 & 0.09 \\
Fap $_{2}$ & $S T_{5}$ & 0.20 & 0.27 \\
\hline
\end{tabular}

interactions among parameters are significant, the sum of the first order indices is less than one, and the pie chart does not cover the $100 \%$ of the total output variance. In contrast, the sum of the total effects can be greater than one, because for example the factor $S_{15}$ contributes to both $S T_{5}$ and $S T_{1}$, hence the percentages in the pie chart of total effects are redistributed according to the value of their sum.

\section{Conclusions and future work}

This research presents a pragmatic approach for estimating the stochastic dynamic response of assembled structures whose interfaces are nonlinear and uncertain. Non-intrusive, or sample-based, UQ has received more and more attention in the engineering community during the last decade and is a very wide and active field of research. The novelty of this work is in incorporating in the UQ process the NL SDMM, which is presented as a reliable and efficient method to avoid the computational burden due to nonlinearities when they can be considered localized and then modeled through lumped elements.

The main issue regarding SDMM, in both linear and nonlinear analyses, is whether the baseline modal base, obtained by using the FE Model, can be updated correctly when the modified local impedance matrix is introduced, or not. The method is more sensitive to the magnitude of the local increments (stiffness, damping, mass) than to the linearity of the modifications. Hence, we consider that free-play behavior is particularly interesting: disruptive changes of local stiffness are considered. A numerical implemen- tation of the SDMM takes care of a reliable modal truncation and eventually to enrich the modal base with specific residual vectors. These are the static vectors of the dofs involved into the modification and can be calculated by applying a unit force on them.

Focus is also put on the similitude between the Sobol's HDMR and the PCE, which allows to obtain the sensitivity indices directly from the coefficients of the expansion. Some theoretical aspects of the key items, i.e., NL SDMM, PCE and GSA, are presented.

The feasibility of the proposed approach is tested on an industrial application. An aircraft component whose dynamic behavior is affected by uncertain free-play junctions is considered and its nonlinear time-domain response is evaluated when it is loaded by a stochastic force. In particular, the root mean square values of the reactions forces at interfaces are selected as QoIs. GSA is proposed as a reliable tool to post-process the outcomes, quantify the effect of each uncertain variable on the QoIs, and select the most relevant ones. The results are discussed looking at their physical coherence and some perspective on the use of PCE in the case of nonlinear problems is given.

The efficiency and predictiveness of meta-models for nonlinear models is an open field of research and further investigations will compare the PCE with other surrogates, such as those based on Radial Basis Functions (RBFs).

\section{Conflict of interest statement}

The authors have no conflict of interest to declare.

\section{References}

[1] S. Hernández, E. Menga, S. Moledo, L. Romera, A. Baldomir, C. López, M.C. Montoya, Optimization approach for identification of dynamic parameters of localized joints of aircraft assembled structures, Aerosp. Sci. Technol. 69 (2017) 538-549.

[2] S. Tol, et al., Dynamic characterization of bolted joints using frf decoupling and optimization, Mech. Syst. Signal Process. 54 (2015) 124-138.

[3] S. Bograd, P. Reuss, A. Schmidt, L. Gaul, M. Mayer, Modeling the dynamics of mechanical joints, Mech. Syst. Signal Process. 25 (8) (2011) 2801-2826.

[4] R. Ibrahim, C. Pettit, Uncertainties and dynamic problems of bolted joints and other fasteners, J. Sound Vib. 279 (3-5) (2005) 857-936.

[5] S. Gangadharan, E. Nikoladaidis, Probabilistic system identification of two flexible joint models, AIAA J. 29 (8) (1991) 1319-1326.

[6] H. Walther, L. Kmetyk, Bolted joints: Model uncertainty vs. test variability, in: Proceedings of the 22nd IMAC, Dearborn, Michigan, (USA), 2004.

[7] P. Kladitis, V. Bright, J. Kharoufeh, Uncertainty in manufacture and assembly of multipe-joint solder self-assembled microelectromechanical systems (mems), J. Manuf. Process. 6 (1) (2004) 32-50.

[8] K. Sommerwerk, B. Michels, K. Lindhorst, M. Haupt, P. Horst, Application of efficient surrogate modeling to aeroelastic analyses of an aircraft wing, Aerosp. Sci. Technol. 55 (2016) 314-323. 
[9] C. Lu, Y.-W. Feng, R.P. Liem, C.-W. Fei, Improved kriging with extremum response surface method for structural dynamic reliability and sensitivity analyses, Aerosp. Sci. Technol. 76 (2018) 164-175

[10] A. Sestieri, Structural dynamic modification, Sadhana 25 (3) (2000) 247-259.

[11] M. Naď, Structural dynamic modification of vibrating systems, Appl. Comput. Mech. 1 (1) (2007) 203-214.

[12] T. Kalaycioğlu, H.N. Özgüven, Nonlinear structural modification and nonlinear coupling, Mech. Syst. Signal Process. 46 (2) (2014) 289-306.

[13] T. Kalaycioğlu, H.N. Özgüven, Harmonic response of large engineering structures with nonlinear modifications, in: Proceedings of the 8th International Conference on Structural Dynamics, EURODYN, 2011, pp. 3623-3629.

[14] E. Menga, S. Hernández, S. Moledo, C. López, Nonlinear dynamic analysis of assembled aircraft structures with concentrated nonlinearities, in: 16th International Forum on Aeroelasticity and Structural Dynamics, IFASD, 2015.

[15] S. Hernández, E. Menga, P. Naveira, C. López, A. Baldomir, M. Cid, S. Moledo, D. Freire, Dynamic analysis of assembled structures with nonlinear joints, in: 58th AIAA/ASCE/AHS/ASC Structures, Structural Dynamics, and Materials Conference, Grapevine, Texas, USA, 2017.

[16] S. Hernández, E. Menga, P. Naveira, D. Freire, C. López, M.C. Montoya, S. Moledo, A. Baldomir, Dynamic analysis of assembled aircraft structures considering interfaces with nonlinear behavior, Aerosp. Sci. Technol. 77 (2018) 265-272.
[17] A. Saltelli, M. Ratto, T. Andres, F. Campolongo, J. Cariboni, D. Gatelli, M. Saisana, S. Tarantola, Global Sensitivity Analysis: The Primer, John Wiley \& Sons, 2008.

[18] B. Sudret, Global sensitivity analysis using polynomial chaos expansions, Reliab. Eng. Syst. Saf. 93 (7) (2008) 964-979.

[19] I.M. Sobol, Uniformly distributed sequences with an additional uniform property, USSR Comput. Math. Math. Phys. 16 (5) (1976) 236-242.

[20] F. Pianosi, K. Beven, J. Freer, J.W. Hall, J. Rougier, D.B. Stephenson, T. Wagener, Sensitivity analysis of environmental models: a systematic review with practical workflow, Environ. Model. Softw. 79 (2016) 214-232.

[21] N. Wiener, The homogoeneous chaos, Am. J. Math. 60 (4) (1938) 897-936.

[22] R. Askey, J. Wilson, Some Basic Hypergeometric Polynomials that Generalize Jacobi Polynomials, Mem. Am. Math. Soc., vol. 319, American Mathematical Society, Providence, RI, 1985, pp. 197-236.

[23] D. Xiu, G.E. Karniadakis, The Wiener-Askey polynomial chaos for stochastic differential equations, SIAM J. Sci. Comput. 187 (2003) 137-167.

[24] S. Hosder, R. Walters, M. Balch, Efficient sampling for non-intrusive polynomial chaos applications with multiple uncertain input variables, in: Proceedings of the 48th AIAA/ASME/ASCE/AHS/ASC Structures, Structural Dynamics, and Materials Conference, Honolulu, HI, 2007.

[25] I.M. Sobol, Theorems and examples on high dimensional model representation, Reliab. Eng. Syst. Saf. 79 (2) (2003) 187-193. 